\title{
SUR UNE $q$-DÉFORMATION LOCALE DE LA THÉORIE DE HODGE NON-ABÉLIENNE EN CARACTÉRISTIQUE POSITIVE
}

\author{
MICHEL GROS
}

\begin{abstract}
RÉsumé. Pour $p$ un nombre premier et $q$ une racine $p$-ième non triviale de 1 , nous présentons les principales étapes de la construction d'une $q$-déformation locale de la "correspondance de Simpson en caractéristique $p$ " dégagée par Ogus et Vologodsky en 2005. La construction est basée sur l'équivalence de Morita entre un anneau d'opérateurs différentiels $q$-déformés et son centre. Nous expliquons aussi les liens espérés entre cette construction et celles introduites récemment par Bhatt et Scholze. Pour alléger l'exposition, nous nous limitons au cas de la dimension 1.
\end{abstract}

For $p$ a prime number and $q$ a non trivial $p$ th root of 1 , we present the main steps of the construction of a local $q$-deformation of the "Simpson correspondence in characteristic $p$ " found by Ogus and Vologodsky in 2005. The construction is based on the Morita-equivalence between a ring of $q$-twisted differential operators and its center. We also explain the expected relations between this construction and those recently done by Bhatt and Scholze. For the sake of readability, we limit ourselves to the case of dimension 1.

TABLE DES MATIÈRES

1. Introduction

2. Rappels sur la théorie d'Ogus et Vologodsky 3

3. Opérateurs différentiels $q$-déformés 6

4. $p$-courbure et Frobenius divisé $q$-déformés 8

5. Théorie de Hodge non-abélienne $q$-déformée 10

6. Questions-Travaux en cours 11

Références 14

\section{INTRODUCTION}

1.1. Ogus et Vologodsky ont dégagé dans [13] un analogue en caractéristique $p>0$ de la théorie de Hodge non-abélienne, i.e. de la correspondance de Simpson complexe. Soient $\tilde{S}$ un schéma plat sur $\mathbb{Z} / p^{2}, \tilde{X}, \tilde{X}^{\prime}$ deux $\tilde{S}$-schémas lisses de réduction modulo $p$ notées $X$ et $X^{\prime}$, et $\tilde{\mathrm{F}}: \tilde{X} \rightarrow \tilde{X}^{\prime}$ un $\tilde{S}$-morphisme. Supposons que ces données constituent un relèvement au-dessus de $\tilde{S}$ du morphisme de Frobenius relatif $\mathrm{F}_{X / S}: X \rightarrow X^{\prime}$ associé à $X$ vu comme schéma au-dessus de $S=\tilde{S} \times_{\mathbb{Z} / p^{2}} \mathbb{Z} / p$. Elles permettent alors à Ogus et Vologodsky d'étendre ([13], Thm. 2.8, Thm. 2.26) aux $\mathscr{O}_{X}$-modules munis d'une connexion intégrable dont la $p$-courbure est supposée seulement quasi-nilpotente à la fois le théorème de descente de Cartier ([10], Thm. 5.1) et l'existence d'une décomposition du complexe de De Rham obtenu par Deligne et Illusie ([5], Rem. 2.2(ii)) induisant l'opération de Cartier ([10], Thm. 7.2). L'exposé oral d'A. Abbes et le nôtre ont été consacrés aux travaux 


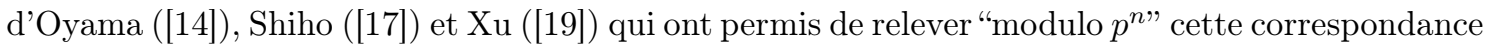
d'Ogus et Vologodsky. C'est ici une autre direction qui est explorée.

1.2. Sans rapport avec ce qui précède, Bhatt, Morrow et Scholze ont dégagé ([3], Thm. 1.8) un raffinement entier des théorèmes standards de comparaison entre cohomologies cristalline, de De Rham et étale $p$-adique pour un schéma formel propre et lisse sur l'anneau des entiers d'une extension non-archimédienne algébriquement close de $\mathbb{C}_{p}$. Dans l'élaboration de celui-ci apparait un relèvement de l'isomorphisme de Cartier ([3], Thm. 8.3) sur la cohomologie d'un objet ([3], Def. 8.1) d'une certaine catégorie dérivée. Dans des situations géométriques locales bien adaptées ([3], 8.5) auxquelles les auteurs se ramènent pour établir cet isomorphisme, l'existence de ce relèvement découle de l'étude de certains $q$-complexes de De Rham ([3], 7.7) avec $q$ une racine $p$-ième non triviale de l'unité dans $\mathbb{C}_{p}$. Ces derniers sont de vrais complexes qui "réalisent" ([3], §8) les objets des catégories dérivées évoquées ci-dessus. Ils ont eux-aussi une cohomologie se calculant par un relèvement de l'opération de Cartier ([16], Prop. 3.4, (iii) ; voir aussi [15], Prop. 2.8) qui explique donc localement l'existence de la précédente. Il nous semble plausible que l'extension espérée du théorème de comparaison entier ([3], Thm. 1.8) à des coefficients non constants ([18]) donne quelque intérêt à essayer d'expliciter une q-déformation locale de la théorie de Hodge non-abélienne incluant l'étude de ce type de complexes et les propriétés de leurs cohomologies. Cela devrait peut-être éclaircir un peu d'éventuels liens entre les théories [13] et [3] puis, ultérieurement, ceux avec la correspondance de Simpson $p$-adique ([1]).

1.3. Le but de ce rapport est d'esquisser, dans ces situations géométriques locales bien adaptées, une telle variante. Les deux résultas principaux sont, d'une part une $q$-déformation locale de la correspondance développée par Ogus-Vologodsky (2.5) et, d'autre part, sa compatibilité aux cohomologies naturelles du but et de la source (5.6). Un corollaire facile (5.7) de notre résultat est l'existence de l'opération de Cartier "relevée" ([16], Prop. 3.4; voir aussi [15], 2.2). Dans le type de situation géométrique que nous considérons, les résultats principaux d'Ogus-Vologodsky qui nous intéressent ici découlent immédiatement d'une équivalence de Morita, à savoir celle associée à la neutralisation d'une algèbre d'opérateurs différentiels vue comme algèbre d'Azumaya sur son centre ([13], Thm. 2.11). Nous développons simplement un $q$-analogue de tout le tableau. Plusieurs choix doivent être faits lors des constructions et il est peu probable que ces résultats puissent se globaliser par les techniques standards, purement schématiques, de recollement (voir par exemple [16], Conj. 1.1 et infra, [15], 2.2 et 3.4 pour une discussion de problèmes analogues, [3], Rem. 8.4).

1.4. Pour pallier ces difficultés de globalisation des q-complexes de De Rham, Bhatt et Scholze ont introduit très récemment dans [4], à beaucoup d'autres fins aussi (dont celle de réinterpréter les théorèmes de comparaison entiers évoqués plus haut ainsi que les décompositions de HodgeTate, ...), de nouvelles techniques, en particulier celles du site prismatique et du site $q$-cristallin. Ils utilisent pour ce faire la théorie des $\delta$-anneaux et leurs avancées fournissent pour nous un espoir de montrer l'indépendance de tout choix auxiliaire (en particulier d'une coordonnée) dans nos constructions, au moins à isomorphisme près. De toute façon, vu la généralité du cadre dans lequel ils se placent et le potentiel d'applications, il nous a paru indispensable d'en tenir compte et de reconsidérer avec leurs nouveaux outils les questions que nous nous posions au moment de la conférence et de la première version de cet article puis d'indiquer les progrès réalisés depuis lors.

1.5. Ce rapport ne contient pas de démonstrations, pour lesquelles on renvoie à [6] et à [8]. Nous insistons plutôt ici sur la mise en parallèle des théories modulo $p$ ([6]) et $q$-déformées ([8]) en spécialisant cette dernière au cadre familier (notations, hypothèses, terminologie, ...) de la théorie de Hodge $p$-adique, ce qui en allège très largement la présentation. Nous résumons très succintement 
tout d'abord au $§ 2$ les principales étapes suivies dans [6] pour établir la neutralisation (loc. cit., Thm. 4.13) mentionnée ci-dessus. La seule nouveauté par rapport à [6] est le résultat de comparaison cohomologique 2.9. Nous passons ensuite au §3, après avoir précisé le cadre géométrique, à la définition des opérateurs différentiels q-déformés. Bien qu'on puisse les définir plus directement (cf. 3.9 ), c'est par un processus de dualité et donc via la définition de parties principales $q$-déformées que nous procédons afin de pouvoir raisonner comme dans la théorie modulo $p$. Dans le $\S 4$, nous déterminons le centre de l'algèbre des opérateurs différentiels $q$-déformés et montrons comment on peut diviser l'action induite par le "Frobenius" (4.6) sur les modules de parties principales $q$-déformées. Que ceci soit possible est pour l'instant l'aspect le plus miraculeux de toute cette théorie. Nous en déduisons enfin la neutralisation (4.11.1) d'une complétion centrale de l'algèbre des opérateurs différentiels $q$-déformés. Le $§ 5$ reformule alors l'équivalence de Morita standard qu'on déduit de cette neutralisation en termes de modules munis d'une $q$-dérivation quasi-nilpotente et de modules de Higgs quasi-nilpotents (2.5) et les conséquences cohomologiques (5.6). Nous terminons enfin au $\S 6$ par quelques observations et questions en relation avec [4].

1.6. Ces résultats sont le fruit d'une collaboration avec B. Le Stum et A. Quirós que l'auteur dégage de toute responsabilité pour les erreurs ou imprécisions qui pourraient apparaitre. L'auteur remercie très sincèrement la Fondation Simons et les organisateurs de la session Simons Symposium on p-adic Hodge Theory (8-12 Mai 2017), Bhargav Bhatt et Martin Olsson, de lui avoir donné l'opportunité d'avancer sur toutes les questions soulevées par ce projet.

\section{Rappels Sur la théorie D'Ogus et Vologodsky}

2.1. Nous conservons dans cette section les notations et hypothèses de 1.1 résumées par les deux diagrammes suivants
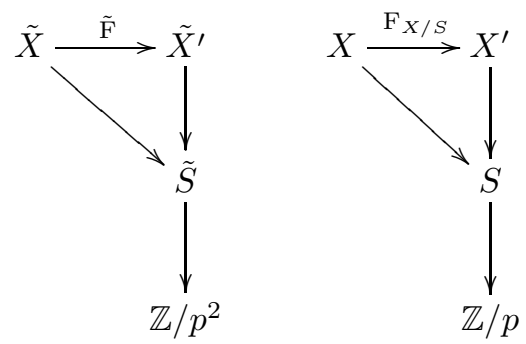

dont celui de droite est donc la réduction modulo $p$ de celui de gauche. Dans ce qui suit, nous allégerons la notation $\mathrm{F}_{X / S}$ en simplement $\mathrm{F}$ mais en attirant bien l'attention du lecteur sur le fait que cet allègement n'est pas tout à fait compatible avec les notations adoptées dans [6] (dans loc. cit., $\mathrm{F}$ est noté $F_{X}$ et $F$ y désigne le Frobenius absolu de $X$ ). Nous supposerons de plus $S$ noethérien pour raccourcir la preuve de 2.9 .

2.2. Pour alléger les notations, nous noterons ici simplement $\mathscr{D}_{X}$ la $\mathscr{O}_{X}$-algèbre $\mathscr{D}_{X}^{(0)}$ des opérateurs différentiels de $X / S$ de niveau $m=0$ introduite par Berthelot [2], 2.2.1 et utilisée dans [6], Def. 2.5, parfois dénommée algèbre des opérateurs PD-différentiels ou algèbre des opérateurs différentiels cristallins. Elle est engendrée par $\mathscr{O}_{X}$ et par les $S$-dérivations de $\mathscr{O}_{X}$ (cf. [2], p. 218, Rem. (i)). Nous noterons Z Z $\mathscr{D}_{X}$ (resp. Z $\mathscr{O}_{X}$ ) le centre de $\mathscr{D}_{X}$ (resp. le centralisateur dans $\mathscr{D}_{X}$ de sa sous-algèbre $\left.\mathscr{O}_{X}\right)$. Nous noterons enfin $\mathrm{S}\left(\mathscr{T}_{X^{\prime}}\right)$ la $\mathscr{O}_{X^{\prime}}$-algèbre (graduée) symétrique du $\mathscr{O}_{X^{\prime-}}$ module $\mathscr{T}_{X^{\prime}}$ des fonctions sur le fibré cotangent de $X^{\prime} / S$. L'application de $p$-courbure permet (cf. 
par exemple [6], Prop. 3.6) de construire un isomorphisme de $\mathscr{O}_{X}$-algèbres

$$
c: \mathrm{S}\left(\mathscr{T}_{X^{\prime}}\right) \stackrel{\sim}{\rightarrow} \mathrm{F}_{*} \mathrm{Z} \mathscr{D}_{X} ; D \in \mathscr{T}_{X^{\prime}} \mapsto D^{p}-D^{[p]} .
$$

On peut, de même (cf. loc. cit.), identifier $\mathrm{Z} \mathscr{O}_{X}$ à $\mathrm{F}^{*} \mathrm{~S}\left(\mathscr{T}_{X^{\prime}}\right)=\mathscr{O}_{X} \otimes_{\mathscr{O}_{X^{\prime}}} \mathrm{S}\left(\mathscr{T}_{X^{\prime}}\right)$.

2.3. L'algèbre $\mathscr{D}_{X}$ agit de manière naturelle de façon $\mathscr{O}_{X^{\prime}}$-linéaire sur $\mathscr{O}_{X}$. Soit $\mathscr{K}_{X}$ le noyau de la surjection canonique $\mathscr{D}_{X} \rightarrow \mathscr{E} n d_{\mathscr{O}_{X^{\prime}}}\left(\mathscr{O}_{X}\right)$. C'est un idéal bilatère de $\mathscr{D}_{X}$. Nous noterons $\widehat{\mathscr{D}_{X}}$ (resp. $\widehat{Z \mathscr{D}_{X}}$, resp. $\widehat{\mathrm{S}\left(\mathscr{T}_{X^{\prime}}\right)}$, resp. $\widehat{\mathrm{Z} \mathscr{O}_{X}}$, resp. $\left.\mathscr{O}_{X} \otimes_{\mathscr{O}_{X^{\prime}}} \widehat{\mathrm{S}\left(\mathscr{T}_{X^{\prime}}\right)}\right)$ le complété adique de $\mathscr{D}_{X}$ (resp. $\mathrm{Z} \mathscr{D}_{X}$, resp. $\mathrm{S}\left(\mathscr{T}_{X^{\prime}}\right)$, resp. Z $\mathscr{O}_{X}$, resp. $\mathscr{O}_{X} \otimes_{\mathscr{O}_{X}} \mathrm{~S}\left(\mathscr{T}_{X^{\prime}}\right)$ ) relativement à l'idéal bilatère $\mathscr{K}_{X}$ (resp. $\mathscr{K}_{X} \cap \mathrm{S}\left(\mathscr{T}_{X^{\prime}}\right)$, resp. $\mathscr{K}_{X} \cap \mathrm{Z} \mathscr{O}_{X}$, resp. $\left.\mathscr{K}_{X} \cap\left(\mathscr{O}_{X} \otimes_{\mathscr{O}_{X}}, \mathrm{~S}\left(\mathscr{T}_{X^{\prime}}\right)\right)\right)$.

2.4. Dans cette situation restrictive d'existence de $\tilde{F}$, plusieurs des résultats généraux de [13] (e.g. Thm. 2.8) découlent immédiatement du résultat suivant ([6], Thm. 4.13) que nous avons appris de P. Berthelot et dont nous rappelerons brièvement le principe de preuve ci-dessous $(2.11,2.12)$.

Théorème 2.5. Toute donnée de $\left(\tilde{X}, \tilde{X}^{\prime}, \tilde{\mathrm{F}}: \tilde{X} \rightarrow \tilde{X}^{\prime}\right)$ comme précédemment définit canoniquement un isomorphisme de $\mathscr{O}_{K}$-algèbres

$$
\widehat{\mathscr{D} X} \stackrel{\sim}{\rightarrow} \mathscr{E} n d_{\widehat{S}\left(\mathscr{T}_{X^{\prime}}\right)}\left(\mathscr{O}_{X} \otimes_{\mathscr{O}_{X^{\prime}}} \widehat{\mathrm{S}\left(\mathscr{T}_{X^{\prime}}\right)}\right) .
$$

On remarquera, en prévision de (4.7), que le but de (2.5.1) est simplement $\mathscr{E}_{n d_{\overparen{\mathrm{Z} \mathscr{X}}}}\left(\widehat{\mathrm{Z} \mathscr{O}_{X}}\right)$.

2.6. Un lemme classique d'algèbre linéaire ([6], Lem. 5.6) montre alors que les anneaux $\widehat{\mathscr{D}_{X}}$ et $\widehat{\mathrm{S}\left(\mathscr{T}_{X^{\prime}}\right)}$ sont, d'une manière complètement explicite, équivalents au sens de Morita : les deux foncteurs suivants entre les catégories de modules sur ces anneaux, $\operatorname{Mod}(\widehat{\mathscr{D} X})$ et $\operatorname{Mod}\left(\widehat{\mathrm{S}\left(\mathscr{T}_{X^{\prime}}\right)}\right)$, correspondantes sont quasi-inverses l'un de l'autre

$$
\begin{aligned}
& \mathbb{H}: \operatorname{Mod}\left(\widehat{\mathscr{D}_{X}}\right) \rightarrow \operatorname{Mod}\left(\widehat{\mathrm{S}\left(\mathscr{T}_{X^{\prime}}\right)}\right) ; \mathscr{E} \mapsto \mathscr{H} o m_{\widehat{\mathscr{D}_{X}}}\left(\mathrm{~F}^{*} \widehat{\mathrm{S}\left(\widehat{\mathscr{T}_{X^{\prime}}}\right)}, \mathscr{E}\right), \\
& \mathbb{M}: \operatorname{Mod}\left(\widehat{\mathrm{S}\left(\mathscr{T}_{X^{\prime}}\right)}\right) \rightarrow \operatorname{Mod}\left(\widehat{\mathscr{D}_{X}}\right) ; \mathscr{F} \mapsto \mathscr{F} \otimes_{\widehat{\mathrm{S}\left(\mathscr{T}_{X^{\prime}}\right)}} \mathrm{F}^{*} \widehat{\mathrm{S}\left(\mathscr{T}_{X^{\prime}}\right)} .
\end{aligned}
$$

Ce résultat fournit, une fois réinterprété (cf. [6], Prop. 5.2) les objets de ces catégories le résultat suivant

Théorème 2.7. Toute donnée de $\left(\tilde{X}, \tilde{X}^{\prime}, \tilde{\mathrm{F}}: \tilde{X} \rightarrow \tilde{X}^{\prime}\right)$ comme précédemment définit canoniquement une équivalence entre la catégorie des $\mathscr{O}_{X}$-modules munis d'une connexion intégrable de p-courbure quasi-nilpotente (cf. [6], Prop. 5.5) et la catégorie des $\mathscr{O}_{X^{\prime}}$-modules munis d'un champ de Higgs quasi-nilpotent (cf. [6], Prop. 5.4).

On vérifie que dans cette équivalence $\mathscr{O}_{X}$ muni de sa connexion canonique $d$ correspond à $\mathscr{O}_{X^{\prime}}$ muni du champ de Higgs nul.

2.8. Le complexe de Higgs d'un $\mathscr{O}_{X^{\prime}}$-module de Higgs $\mathscr{F} \in \operatorname{Mod}\left(\widehat{\mathrm{S}\left(\mathscr{T}_{X^{\prime}}\right)}\right)$ est, par définition, le complexe (avec $\mathscr{F}$ placé en degré 0)

$$
0 \rightarrow \mathscr{F} \stackrel{\theta}{\longrightarrow} \mathscr{F} \otimes_{\mathscr{O}_{X^{\prime}}} \Omega_{X^{\prime}}^{1} \stackrel{(-) \wedge \theta}{\longrightarrow} \mathscr{F} \otimes_{\mathscr{O}_{X^{\prime}}} \Omega_{X^{\prime}}^{2} \stackrel{(-) \wedge \theta}{\longrightarrow} \ldots
$$

avec $\theta$ l'application $\mathscr{O}_{X^{\prime}}$-linéaire provenant de la structure naturelle de $\mathrm{S}\left(\mathscr{T}_{X^{\prime}}\right)$-module sur $\mathscr{F}$ et, pour alléger, $\Omega_{X^{\prime}}^{i}$ le $\mathscr{O}_{S^{-}}$-module des différentielles relatives de de degré $i$ de $X^{\prime} / S$ (noté $\Omega_{X^{\prime} / S}^{i}$ lorsque une ambiguïté est possible). On en donnera ci-dessous (2.9.4) une autre description. Il résulte facilement de cette équivalence la 
Proposition 2.9. Si $\mathscr{E} \in \operatorname{Mod}\left(\widehat{\mathscr{D}_{X}}\right)$ et $\mathscr{F} \in \operatorname{Mod}\left(\widehat{\left.\mathrm{S}_{\left(X^{\prime}\right.}\right)}\right)$ se correspondent par l'équivalence cidessus, alors l'image directe par $\mathrm{F}$ du complexe de De Rham de $\mathscr{E}$ est quasi-isomorphe au complexe de Higgs de $\mathscr{F}$.

Le principe de démonstration est le suivant. Pour calculer $\mathbb{R} \mathscr{H}_{0 m_{\mathscr{D}_{X}}}\left(\mathscr{O}_{X}, \mathscr{E}\right)$, on utilise la résolution de Spencer de $\mathscr{O}_{X}$ par des $\mathscr{O}_{X}$-localement libres sur $\mathscr{D}_{X}$

$$
\left[\ldots \rightarrow \mathscr{D}_{X}^{(0)} \otimes_{\mathscr{O}_{X}} \wedge^{2} \mathscr{T}_{X} \rightarrow \mathscr{D}_{X}^{(0)} \otimes_{\mathscr{O}_{X}} \mathscr{T}_{X} \rightarrow \mathscr{D}_{X}\right] \rightarrow \mathscr{O}_{X} \rightarrow 0 .
$$

On tensorise alors la partie entre crochets par $\widehat{\mathscr{D}_{X}}$ en préservant l'exactitude de 2.9 .1 car $\widehat{\mathscr{D}_{X}}$ est plat sur $\mathscr{D}_{X}$ puisque c'est le complété de $\mathscr{D}_{X}$ relativement à un idéal bilatère engendré par une suite centralisante. On a alors, notant $\Omega_{X}^{\bullet}$ pour alléger le complexe $\Omega_{X / S}^{\bullet}$ des différentielles relatives de $X / S$, des isomorphismes

$$
\mathbb{R} \mathscr{H}_{0 m_{\mathscr{D}_{X}}}\left(\mathscr{O}_{X}, \mathscr{E}\right) \simeq \mathscr{H}_{o m}\left(\widehat{\mathscr{D}_{X}} \otimes_{\mathscr{O}_{X}} \wedge \mathscr{T}_{X}, \mathscr{E}\right) \simeq \mathscr{E} \otimes_{\mathscr{O}_{X}} \Omega_{X}^{\bullet}
$$

Pour le complexe de Higgs, on utilise la résolution de Koszul de $\mathscr{O}_{X^{\prime}}$

$$
\left[\ldots \rightarrow \mathrm{S}\left(\mathscr{T}_{X^{\prime}}\right) \otimes_{\mathscr{O}_{X^{\prime}}} \wedge^{2} \mathscr{T}_{X^{\prime}} \rightarrow \mathrm{S}\left(\mathscr{T}_{X^{\prime}}\right) \otimes_{\mathscr{O}_{X^{\prime}}} \mathscr{T}_{X^{\prime}} \rightarrow \mathrm{S}\left(\mathscr{T}_{X^{\prime}}\right)\right] \rightarrow \mathscr{O}_{X^{\prime}} \rightarrow 0 .
$$

que l'on tensorise par $\widehat{\mathrm{S}\left(\mathscr{T}_{X^{\prime}}\right)}$ au-dessus de $\mathrm{S}\left(\mathscr{T}_{X^{\prime}}\right)$ en la laissant exacte. On obtient

$$
\mathbb{R} \mathscr{H} \text { om }_{\mathrm{S}\left(\mathscr{T}_{X^{\prime}}\right)}\left(\mathscr{O}_{X^{\prime}}, \mathscr{F}\right) \simeq \mathscr{H}_{o m_{\mathrm{S}}\left(\widehat{\left.\mathscr{T}_{X^{\prime}}\right)}\right.}\left(\widehat{\mathrm{S}\left(\mathscr{T}_{X^{\prime}}\right)} \otimes_{\mathscr{O}_{X^{\prime}}} \wedge \cdot \mathscr{T}_{X^{\prime}}, \mathscr{F}\right) \simeq \mathscr{F} \otimes_{\mathscr{O}_{X^{\prime}}} \Omega_{X^{\prime}}^{\bullet}
$$

Les deux foncteurs dérivés 2.9.2 et 2.9.4 pouvant se calculer à l'aide de résolutions injectives du second argument, la proposition s'ensuit grâce à l'équivalence de catégories donnée par $\mathbb{H}$ et $\mathbb{M}$.

2.10. Notons ici que, par définition de $\mathscr{K}_{X}$ et grâce au lemme d'algèbre linéaire qu'on vient d'évoquer, les anneaux $\mathscr{D}_{X} / \mathscr{K}_{X}$ et $\mathscr{O}_{X^{\prime}}$ sont équivalents au sens de Morita : c'est, réinterprété dans ce langage, le classique théorème de descente de Cartier ([10], Thm. 7.2). D'autre part, la proposition 2.9 fournit exactement, une fois précisé les isomorphismes, la décomposition du complexe de De Rham obtenue par Deligne-Illusie ([5], Rem. 2.2(ii)).

2.11. La démonstration du théorème 2.5 procède par dualité. Soient $\mathscr{I} \subset \mathscr{O}_{X \times_{S} X}$ l'idéal définissant l'immersion diagonale $X \hookrightarrow X \times_{S} X, \mathscr{P}_{X}$ son enveloppe à puissances divisées (notée $\mathscr{P}_{X / S,(0)}$ dans [6], 2.4), $\overline{\mathscr{I}} \subset \mathscr{P}_{X}$ le $P D$-idéal engendré par $\mathscr{I}$ et, pour $n$ un entier $\geq 0, \mathscr{P}_{X}^{n}=\mathscr{P}_{X} / \mathscr{I}^{[n+1]}$. On a, par définition,

$$
\mathscr{D}_{X, n}=\mathscr{H}_{o m_{\mathscr{O}_{X}}}\left(\mathscr{P}_{X}^{n}, \mathscr{O}_{X}\right) ; \mathscr{D}_{X}=\cup_{n \geq 0} \mathscr{D}_{X, n}
$$

On remarque alors qu'on a simplement un isomorphisme

$$
\widehat{\mathscr{D}_{X}} \stackrel{\sim}{\rightarrow} \mathscr{H}_{o m_{\mathscr{O}_{X}}}\left(\mathscr{P}_{X}, \mathscr{O}_{X}\right)
$$

et que $\widehat{\mathscr{D}_{X}}$ n'est autre que ce qui est classiquement appelé l'algèbre des opérateurs hyper- $P D$ différentiels. D'autre part, notons $\Gamma\left(\Omega_{X^{\prime}}^{1}\right)$ la $\mathscr{O}_{X^{\prime}}$-algèbre (graduée) à puissances divisées canoniquement associée au $\mathscr{O}_{X^{\prime}}$-module $\Omega_{X}^{1}$ ([6], Thm. 1.2). Une vérification d'algèbre linéaire ([6], preuve de Thm. 4.13) fournit un isomorphisme

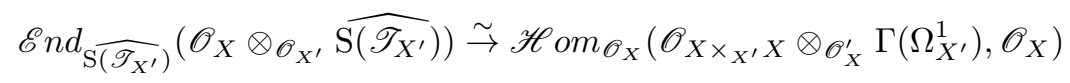

de sorte que le théorème 2.5 se réduit à la construction d'un isomorphisme d'algèbres de Hopf

$$
\mathscr{O}_{X \times_{X^{\prime}} X} \otimes_{\mathscr{O}_{X^{\prime}}} \Gamma\left(\Omega_{X^{\prime}}^{1}\right) \stackrel{\sim}{\rightarrow} \mathscr{P}_{X} .
$$

2.12. Cette construction procède selon les principales étapes suivantes : 
2.12.1. L'application canonique $\mathscr{I} \rightarrow \mathscr{P}_{X} ; f \rightarrow f^{[p]}$ composée avec la projection canonique $\mathscr{P}_{X} \rightarrow \mathscr{I} \mathscr{P}_{X}$ est une application $\mathrm{F}^{*}$-linéaire nulle sur $\mathscr{I}^{2}$ ([6], Lem. 3.1). Elle induit donc ([6], Prop. 3.2) par passage au quotient et linéarisation une application $\mathscr{O}_{X}$-linéaire

$$
\mathrm{F}^{*} \Omega_{X^{\prime}}^{1} \rightarrow \mathscr{P}_{X} / \mathscr{I} \mathscr{P}_{X}
$$

2.12.2. L'application (2.12.1) s'étend en un isomorphisme de $\mathscr{O}_{X}$-algèbres à puissances divisées ([6], Prop. 3.3)

$$
\mathrm{F}^{*} \Gamma\left(\Omega_{X^{\prime}}^{1}\right) \stackrel{\sim}{\rightarrow} \mathscr{P}_{X} / \mathscr{I} \mathscr{P}_{X}
$$

2.12.3. La donnée de $\left(\tilde{X}, \tilde{X}^{\prime}, \tilde{\mathrm{F}}: \tilde{X} \rightarrow \tilde{X}^{\prime}\right)$ permet de factoriser le morphisme (2.12.1) en un morphisme de $\mathscr{O}_{X}$-modules

$$
\mathrm{F}^{*} \Omega_{X^{\prime}}^{1} \rightarrow \mathscr{P}_{X}
$$

C'est l'application Frobenius divisé, notée $\frac{1}{p !} \tilde{\mathrm{F}}^{*}$ dans (Prop. 4.8, [6]). On prendra garde ici que la surjection canonique $\mathscr{P}_{X} \rightarrow \mathscr{P}_{X} / \mathscr{I}_{\mathscr{P}_{X}}$ n'est pas compatible aux puissances divisées.

2.12.4. L'application (2.12.3) s'étend en un morphisme de $\mathscr{O}_{X}$-algèbres à puissances divisées ([6], Prop. 4.8)

$$
\mathrm{F}^{*} \Gamma\left(\Omega_{X^{\prime}}^{1}\right) \rightarrow \mathscr{P}_{X}
$$

factorisant l'isomorphisme 2.12 .2 .

2.12.5. L'application (2.12.4) s'étend canoniquement en un isomorphisme de $\mathscr{O}_{X}$-algèbres de Hopf ([6], Prop. 4.13)

$$
\mathscr{O}_{X \times_{X^{\prime}} X} \otimes_{\mathscr{O}_{X^{\prime}}} \mathrm{F}^{*} \Gamma\left(\Omega_{X^{\prime}}^{1}\right) \stackrel{\sim}{\rightarrow} \mathscr{P}_{X} .
$$

C'est l'isomorphisme (2.11.4) recherché.

2.13. Il peut être utile au lecteur de savoir que si l'on composait la projection canonique $\mathscr{P}_{X} \rightarrow$ $\mathscr{P}_{X} / \mathscr{I} \mathscr{P}_{X}$ avec l'inverse de $(2.12 .2)$ et qu'on dualisait l'application obtenue, on retrouverait la composée $\mathrm{S}\left(\mathscr{T}_{X^{\prime}}\right) \rightarrow \mathrm{F}_{*} \mathscr{D}_{X}$ de l'application de $p$-courbure $c$ (2.2.1) et de l'application canonique $\mathrm{Z} \mathscr{D}_{X} \hookrightarrow \mathscr{D}_{X}$

\section{OpÉrateurs DifFÉREnTIELS $q$-DÉFORMÉS}

3.1. Soient $R$ un anneau commutatif supposé muni d'un relèvement qu'on notera ici simplement $\mathrm{F}$ du Frobenius absolu de $R / p$ et $q \in R$. Soient également $A$ une $R$-algèbre munie d'un morphisme étale $f: R[t] \rightarrow A$ (i.e. d'un framing au sens de $[16], \S 3 ;[3], \S 8, \ldots$ ). On munit $R[t]$ des deux morphismes de $R$-algèbres $\sigma$ et $\mathrm{F}^{*}$ induits par $\sigma(t)=q t$ et $\mathrm{F}^{*}(t)=t^{p}$. On supposera également, par simplicité, qu'il existe deux morphismes de $R$-algèbres notés encore $\sigma: A \rightarrow A$ et $\mathrm{F}^{*}: A^{\prime}:=R_{\nwarrow} \mathrm{F} \otimes_{R} A \rightarrow A$, tels que respectivement $\sigma(x)=q x$ et $\mathrm{F}^{*}(1 \otimes x)=x^{p}$ avec $x:=f(t)$ (élément parfois appelé coordonnée sur $A$ ). Signalons immédiatement, pour fixer les idées, un exemple particulièrement intéressant pour nous où une telle situation se manifeste. Soient $\overline{\mathbb{Q}}_{p}$ une clôture algébrique de $\mathbb{Q}_{p}, q \in \overline{\mathbb{Q}}_{p}$ une racine $p$-ième de 1 non triviale, $K$ l'extension finie totalement ramifiée de $\mathbb{Q}_{p}$ engendrée par $q$ et $R:=\mathscr{O}_{K}$ l'anneau des entiers de $K$ muni de $\mathrm{F}=\mathrm{Id}_{\mathrm{R}}$. Alors, la simple donnée de $f$ étale comme ci-dessus et des arguments standards suffisent à produire, par passage à la complétion $p$-adique de $A$, une situation comme précédemment pour cette dernière. 
On s'est limité au cadre de la dimension 1 mais tout ce qui précède et suit vaut en dimension supérieure. On a également fixé une fois pour toutes ce dont on aura besoin mais les données ne seront utilisés qu'au fur et à mesure (la donnée de Frobenius n'est pas requise avant §4).

3.2. Pour $u$ une indéterminée et $n$ un entier $\geq 0$, on pose $(n)_{u}=\frac{u^{n}-1}{u-1} \in \mathbb{Z}[u] ;(n)_{u} !=\prod_{i=1}^{n}(i)_{u} \in$ $\mathbb{Z}[u] ;\left(\begin{array}{c}n \\ k\end{array}\right)_{u}=\frac{(n)_{u} !}{(k)_{u} !(n-k)_{u} !} \in \mathbb{Z}[u]$. Si maintenant $q$ est un élément de $R$ comme dans 3.1 , les notations $(n)_{q} ;(n)_{q} ! ;\left(\begin{array}{c}n \\ k\end{array}\right)_{q}$ signifient qu'on a évalué les quantités précédentes en $u=q$ afin d'obtenir des éléments de $R$. Ayant à éviter plus bas une possible confusion avec la notation standard des puissances divisées par des crochets, nous avons adopté la notation $(n)_{q}$ avec des parenthèses plutôt que la notation $[n]_{q}$ de $([7]$ ou $[16], \S 1)$.

3.3. Soient $A$ comme dans 3.1 et fixons $y \in A$. On va définir tout d'abord l'analogue $q$-déformé des sections de $\mathscr{P}_{X}$ (2.11) sur un ouvert affine dans ce cadre. Soit $A\langle\xi\rangle_{q, y}$ le $A$-module libre de générateurs abstraits notés $\xi^{[n]_{q, y}}$ avec $n \in \mathbb{N}(\mathrm{cf}$. [8], §2). On abrégera, lorsqu'aucune confusion n'en résulte, $\xi^{[0]_{q, y}}$ en 1 et $\xi^{[1]_{q, y}}$ en $\xi$. On notera $I^{[n+1]_{q, y}}$ le sous- $A$-module libre de $A\langle\xi\rangle_{q, y}$ engendré par les $\xi^{[k]_{q, y}}$ avec $k>n$.

Proposition 3.4 ([8], Prop. 2.2). Soient $m, n \in \mathbb{N}$. La règle de multiplication

$$
\xi^{[n]} \cdot \xi^{[m]}=\sum_{i=0}^{\min (m, n)}(-1)^{i} q^{\frac{i(i-1)}{2}}\left(\begin{array}{c}
m+n-i \\
m
\end{array}\right)_{q}\left(\begin{array}{c}
m \\
i
\end{array}\right)_{q} y^{i} \xi^{[m+n-i]}
$$

permet de munir $A\langle\xi\rangle_{q, y}$ une structure de A-algèbre commutative et unitaire. L'ensemble $I^{[n+1]}$ est un idéal de $A\langle\xi\rangle_{q, y}$.

On dira alors que $A\langle\xi\rangle_{q, y}$ est l'anneau des polynômes sur $A$ à puissances divisées q-déformées.

Cette terminologie est justifiée par l'égalité, valide pour tout $n \in \mathbb{N}$,

$$
(n)_{q} ! \xi^{[n]}=\prod_{i=0}^{n-1}\left(\xi+(i)_{q} y\right)=: \xi^{(n)}
$$

et le fait que les $\xi^{(n)}$ forment, pour $n \in \mathbb{N}$, une base de $A[\xi]$ ([8], Lem. 1.1).

3.5. Soit encore $A$ comme dans 3.1. Supposons désormais que $y=(1-q) x \in A$. Le lecteur remarquera que lorsque $q=1$, l'algèbre $A\langle\xi\rangle_{q, y}$ n'est autre que la $A$-algèbre des polynômes à puissances divisées usuelles en $\xi$.

Définition 3.6 ([8], Def. 4.2). Soit $n \in \mathbb{N}$. Le $A$-module des parties principales q-déformées de $A$ d'ordre au plus $n$ (et de niveau 0) et le $A$-module des parties principales $q$-déformées de $A$ sont, respectivement,

$$
\begin{gathered}
\mathrm{P}_{A / R, \sigma, n}^{(0)}=A\langle\xi\rangle_{q, y} / I^{[n+1]_{q, y}}, \\
\mathrm{P}_{A / R, \sigma}^{(0)}=\lim _{\overleftarrow{n \in \mathbb{N}}} A\langle\xi\rangle_{q, y} / I^{[n+1]_{q, y}} .
\end{gathered}
$$

Dans la suite, nous allégerons les notations $\mathrm{P}_{A / R, \sigma, n}^{(0)}$ et $\mathrm{P}_{A / R, \sigma}^{(0)}$ en $\mathrm{P}_{A, \sigma, n}$ et $\mathrm{P}_{A, \sigma}$ respectivement. 
3.7. On conserve les hypothèses et notations de 3.5. Rappelons qu'il découle de ([12], Prop. 2.10) qu'il existe un unique endomorphisme $R$-linéaire $\partial_{\sigma}$ de $A$ tel que, pour tous $z_{1}, z_{2} \in A$, on ait

$$
\partial_{\sigma}\left(z_{1} z_{2}\right)=z_{1} \partial_{\sigma}\left(z_{2}\right)+\sigma\left(z_{1}\right) \partial_{\sigma}\left(z_{2}\right)
$$

i.e. une $\sigma$-dérivation canonique. Afin de construire les opérateurs différentiels $q$-déformés par dualité, nous aurons besoin de la définition suivante.

Définition 3.8 ([8], Def. 4.5). L'application de Taylor q-déformée (de niveau 0) est l'application

$$
\mathscr{T}: A \rightarrow \mathrm{P}_{A, \sigma}
$$

définie par $\mathscr{T}(z)=\sum_{k=0}^{+\infty} \partial_{\sigma}^{k}(z) \xi^{[k]}$ pour tout $z \in A$.

On peut en fait définir $\mathscr{T}$ de manière plus formelle (cf. [8], Def. 4.5) et vérifier que c'est un morphisme d'anneaux, puis décrire cette application grâce à $\partial_{\sigma}$ comme on vient de le faire.

Si maintenant $M$ est un $A$-module à gauche, l'écriture $\mathrm{P}_{A, \sigma, n} \otimes_{R}^{\prime} M$ signifie que nous regardons $\mathrm{P}_{A, \sigma, n}$ comme un $A$-module via l'application $\mathscr{T}$ (3.8.1). Autrement dit, pour tous $z \in A, s \in M, k \in$ $\mathbb{N}$, on a :

$$
\xi^{[k]} \otimes^{\prime} z s=\mathscr{T}(z) \xi^{[k]} \otimes^{\prime} s
$$

Ceci permet de définir, pour chaque $n \in \mathbb{N}$,

$$
\mathrm{D}_{A, \sigma, n}^{(0)}=\operatorname{Hom}_{A}\left(\mathrm{P}_{A, \sigma, n} \otimes_{A}^{\prime} A, A\right) .
$$

Pour $n \in \mathbb{N}$, ces $A$-modules forment un système inductif et permettent donc de considérer

$$
\mathrm{D}_{A, \sigma}^{(0)}=\underset{n \in \mathbb{N}}{\lim } \mathrm{D}_{A, \sigma, n}^{(0)}
$$

On vérifie alors que la comultiplication

$$
\mathrm{P}_{A, \sigma} \rightarrow \mathrm{P}_{A, \sigma} \otimes_{A}^{\prime} \mathrm{P}_{A, \sigma}
$$

définie par $\xi^{[n]} \mapsto \sum_{i=0}^{n} \xi^{[n-i]} \otimes^{\prime} \xi^{[i]}$ permet de munir $\mathrm{D}_{A, \sigma}^{(0)}$ d'une structure d'anneau (cf. [8], Prop. $5.6)$.

3.9. L'anneau (3.8.4) ainsi construit par dualité n'est autre (cf. [8], Prop. 5.7) que l'extension de Ore $\mathrm{D}_{A / R, \sigma}$ de $A$ par $\sigma$ et $\partial_{\sigma}$, c'est-à-dire le $A$-module libre de générateurs abstraits $\partial_{\sigma}^{k}(k \geq 0)$ avec la règle de commutation $\partial_{\sigma} z=\sigma(z) \partial_{\sigma}+\partial_{\sigma}(z)$ pour tout $z \in A$. Dans la suite, on utilisera cette notation $\mathrm{D}_{A, \sigma}$ pour l'anneau (3.8.4) si aucune confusion n'en résulte.

\section{4. $p$-COURBURE ET Frobenius divisé $q$-DÉFormÉS}

4.1. On garde dans tout ce $\S$ les notations et hypothèses de 3.1 et l'on suppose de plus que $(p)_{q}=0$ dans $R$ et que $R$ est $q$-divisible, c'est-à-dire (cf. [8], 0.3) que pour tout $m \in \mathbb{N},(m)_{q}$ est inversible dans $R$ s'il est non nul. Ces deux conditions (qui ne sont pas nécessaires simultanément dans tous les énoncés) sont réalisés, par exemple, dans le cas où $R=\mathscr{O}_{K}$ (3.1) et $q \neq 1$ une racine $p$-ième de l'unité. On continue de poser $y=(1-q) x$ comme dans 3.5 .

Soient $\mathrm{ZD}_{A, \sigma}$ le centre de l'anneau $\mathrm{D}_{A, \sigma}$ et $\mathrm{Z}_{A, \sigma}$ le centralisateur dans $\mathrm{D}_{A, \sigma}$ de sa sous-algèbre A. 
q-DÉFORMATION DE LA THÉORIE DE HODGE NON-ABÉLIENNE EN CARACTÉRISTIQUE POSITIVE 9

Proposition 4.2 ([8], Prop. 6.3, Def 6.5). Il existe une unique application A-linéaire de A-algèbres

$$
A[\theta] \rightarrow \mathrm{D}_{A, \sigma} ; \theta \mapsto \partial_{\sigma}^{p}
$$

dite de p-courbure q-déformé (ou simplement p-courbure tordue). Elle induit un isomorphisme de $A$-algèbres entre $A[\theta]$ et $\mathrm{Z} A_{A, \sigma}$ et de $A^{\prime}$-algèbres entre $A^{\prime}[\theta]$ et $\mathrm{ZD}_{A, \sigma}$.

Cette application est construite par dualité à partir des applications canoniques $\mathrm{P}_{A, \sigma, n p} \rightarrow$ $\mathrm{P}_{A, \sigma, n p} /(\xi)$.

4.3. L'analogue $q$-déformé du calcul local crucial permettant de prouver l'existence de l'isomorphisme (2.12.2) est l'énoncé suivant.

Proposition 4.4 ([8], Def 2.5, Thm. 2.6). L'unique application A-linéaire

$$
A\langle\omega\rangle_{1, y^{p}} \rightarrow A\langle\xi\rangle_{q, y} ; \omega^{[k]} \mapsto \xi^{[p k]}
$$

est appelée Frobenius divisé q-déformé (ou simplement Frobenius divisé tordu). C'est un homomorphisme d'anneaux induisant un isomorphisme de A-algèbres

$$
A\langle\omega\rangle_{1, y^{p}} \stackrel{\sim}{\rightarrow} A\langle\xi\rangle_{q, y} /(\xi) .
$$

4.5. Pour $n$ et $i$ des entiers $\geq 0$, on définit (cf. [8], Def 7.4, Prop. 7.9) des polynômes $A_{n, i}(u), B_{n, i}(u) \in$ $\mathbb{Z}[u]$ par les formules

$$
A_{n, i}(u):=\sum_{j=0}^{n}(-1)^{n-j} u^{\frac{p(n-j)(n-j-1}{2}}\left(\begin{array}{c}
n \\
j
\end{array}\right)_{u^{p}}\left(\begin{array}{c}
p j \\
i
\end{array}\right)_{u}
$$

et

$$
(n)_{u} ! A_{n, i}(u)=(n)_{u^{p}} !(p)_{u}^{n} B_{n, i}(u)
$$

Les polynômes $A_{n, i}(u)$ s'introduisent naturellement dans la description de l'action induite par $\mathrm{F}$ sur les modules de parties principales $q$-déformées (cf. [8], Prop. 7.5). La possibilité de définir les polynômes $B_{n, i}(u)$ vient, quant à elle, de l'examen des coefficients des $A_{n, i}(u)$.

Proposition 4.6 ([8], Prop. 7.12). L'application

$$
\left[\mathrm{F}^{*}\right]: A^{\prime}\langle\omega\rangle_{1, y} \rightarrow A\langle\xi\rangle_{q, y} ;\left[\mathrm{F}^{*}\right]\left(\omega^{[n]}\right)=\sum_{i=n}^{p n} B_{n, i}(q) x^{p n-i} \xi^{[i]}
$$

est un homomorphisme d'anneaux.

Grâce à cette application, on montre, comme pour 2.12.5 :

Proposition 4.7 ([8], Prop. 7.13). L'application [F*] induit un morphisme de A-algèbres

$$
\left[\mathrm{F}^{*}\right]: A[\xi] /\left(\xi^{(p)_{q, y}}\right) \otimes_{A^{\prime}} \quad A^{\prime}\langle\omega\rangle_{1, y} \stackrel{\sim}{\rightarrow} A\langle\xi\rangle_{q, y},
$$

qui est un isomorphisme.

C'est l'analogue $q$-déformé du calcul local crucial permettant de prouver l'existence de l'isomorphisme (2.12.5) ([6], Thm. 4.13).

4.8. Par dualité, on déduit de 4.7 la q-déformation suivante de ([6], Prop. 4.8).

Proposition 4.9 ([8], Prop. 8.1). L'application [F*] induit, par dualité, un morphisme de Amodules

$$
\Phi_{A, \sigma}: \mathrm{D}_{A, \sigma} \rightarrow \mathrm{Z} A_{A, \sigma} \hookrightarrow \mathrm{D}_{A, \sigma} ; \partial_{\sigma}^{n} \mapsto \sum_{k=0}^{n} B_{k, n}(q) x^{p k-n} \partial_{\sigma}^{p k}
$$


4.10. Soient, respectivement, $\widehat{\mathrm{D}_{A, \sigma}}, \widehat{\mathrm{ZD}_{A, \sigma}}, \widehat{\mathrm{ZA}_{A, \sigma}}$ les complétés adiques de $\mathrm{D}_{A, \sigma}, \mathrm{ZD}_{A, \sigma}, \mathrm{Z}_{A, \sigma}$ (4.1) relativement à l'élément central $\partial_{\sigma}^{p} \in \mathrm{ZD}_{A, \sigma}$.

Théorème 4.11 ([8], Thm. 8.7). L'application $\Phi_{A, \sigma}$ induit un isomorphisme de A-algèbres

$$
\widehat{\mathrm{D}_{A, \sigma}} \stackrel{\sim}{\rightarrow} \operatorname{End}_{\widehat{\mathrm{ZD}_{A, \sigma}}}\left(\widehat{\mathrm{Z} A_{A, \sigma}}\right) \text {. }
$$

4.12. Pour $q=1$, réduisant modulo $p$, cet isomorphisme redonne l'isomorphisme (2.5.1). A un choix de normalisation près (correspondant exactement à la $q$-déformation de la différence entre diviser par $p$ ou par $p$ ! dans la construction du Frobenius divisé en caractéristique $p$ ), pour $A=$ $R[t], f=\mathrm{Id}$, l'isomorphisme (4.11.1) se décrit explicitement comme dans ([7], §4).

\section{Théorie de Hodge nON-ABÉlienne $q$-DÉForméE}

On conserve dans ce $\S$ les hypothèses et notations générales du $\S 4$.

5.1. Le lemme classique d'algèbre linéaire ([6], Lem. 5.6) déjà évoqué en 2.6 montre alors que les anneaux $\widehat{\mathrm{D}_{A, \sigma}}$ et $\widehat{\mathrm{ZD}_{A, \sigma}}$ sont équivalents au sens de Morita. On va traduire cette conséquence en termes plus explicites.

Définition 5.2. Soit $M$ un $A$-module. Une $\sigma$-dérivation (de niveau 0) ou simplement $\sigma$-dérivation de $M$ est une application $R$-linéaire $\partial_{\sigma, M}\left(=: \partial_{\sigma, M}^{<1>0}\right)$ vérifiant, pour tous $r \in A, m \in M$, l'égalité (règle de Leibniz $q$-déformée)

$$
\partial_{\sigma, M}(r m)=\partial_{\sigma}(r) m+\sigma(r) \partial_{\sigma, M}(m) .
$$

On a une notion évidente de morphismes entre modules munis de $\sigma$-dérivations. Rappelons maintenant qu'on dit qu'un endomorphisme $u_{G}$ d'un groupe abélien $G$ est dit quasi-nilpotent si pour tout $g \in G$, il existe $n \in \mathbb{N}$ tel que $u_{G}^{n}(g)=0$.

5.3. Dans le cadre géométrique du $\S 4$, l'analogue $q$-déformé de la correspondance d'Ogus-Vologodsky (2.6.1), (2.6.2) est l'énoncé suivant.

Théorème 5.4 ([8], Cor. 8.9). La catégorie des A-modules $M$ munis d'une $\sigma$-dérivation quasinilpotente $\sigma_{M}$ est équivalente à la catégorie des $A^{\prime}$-modules $H$ munis d'un endomorphisme $A$ linéaire quasi-nilpotent $u_{H}$.

L'équivalence est donnée explicitement (comparer avec [6], Prop. 5.7 pour la situation en caractéristique $p$ ) par les deux foncteurs suivants quasi-inverses l'un de l'autre

$$
\begin{aligned}
\mathbb{H}_{q}:\left(M, \sigma_{M}\right) \mapsto\left(H:=\left\{m \in M \mid \Phi_{A, \sigma}\left(\partial_{\sigma}^{k}\right)(m)=\partial_{\sigma}^{k}(m) \text { pour tout } k \in \mathbb{N}\right\}, \partial_{\sigma}^{p}\right), \\
\mathbb{M}_{q}:\left(H, u_{H}\right) \mapsto\left(M:=A \otimes_{A^{\prime}} N, \partial_{\sigma, M}\right)
\end{aligned}
$$

avec $\partial_{\sigma, M}$ l'unique $\sigma$-dérivation de $M$ telle que $\partial_{\sigma, M}(1 \otimes h)=t^{p-1} \otimes u_{H}(h)$ pour tout $h \in H$. Dans cette équivalence, $\left(A, \partial_{\sigma}\right)(3.7)$ correspond à $\left(A^{\prime}, 0\right)$.

5.5. Formulons maintenant les conséquences cohomologiques de cette équivalence en termes analogues à ceux de 2.9. Si $M$ est un $A$-module muni d'une $\sigma$-dérivation $\partial_{\sigma, M}$, on lui associe son complexe de De Rham $q$-déformé ou, s'inspirant de la terminologie de [16], complexe de q-De Rham de $M$

$$
q-\operatorname{DR}(M / R): 0 \rightarrow M \stackrel{\nabla_{M}}{\longrightarrow} M \otimes_{A} \Omega_{A / R}^{1} \rightarrow 0
$$

avec $M$ placé en degré 0 et $\nabla_{M}(m)=\partial_{\sigma, M}(m) \otimes d x$. Bien que cela ne joue pas de rôle à ce niveau, signalons ici qu'il serait beaucoup plus canonique dans cette définition d'utiliser le $R$-module des 
q-DÉFORMATION DE LA THÉORIE DE HODGE NON-ABÉLIENNE EN CARACTÉRISTIQUE POSITIVE 11

différentielles q-déformées $\Omega_{A / R, \sigma}^{1}$ de [11], Def. 5.3. plutôt que $\Omega_{A / R}^{1}$ (qui lui est seulement noncanoniquement isomorphe).

D'autre part, pour $H$ un $A^{\prime}$-module muni d'un endomorphisme $u_{H}$, on peut lui associer son complexe de Higgs

$$
\operatorname{Higgs}(H / R): 0 \rightarrow H \stackrel{\theta_{H}}{\rightarrow} H \otimes_{A^{\prime}} \Omega_{A^{\prime} / R}^{1} \rightarrow 0
$$

avec $H$ placé en degré 0 et $\theta_{H}(h)=u_{H}(h) \otimes d x$.

Proposition 5.6 ([8], Cor. 8.10). Si $\left(M, \partial_{\sigma, M}\right)$ est un A-module muni d'une dérivation q-déformée quasi-nilpotente et $\left(H, u_{H}\right)$ un $A^{\prime}$-module muni d'un endomorphisme quasi-nilpotent se correspondant suivant les foncteurs $\mathbb{H}_{q}$ et $\mathbb{M}_{q}$, alors le complexe $q-\mathrm{DR}(M / R)$ est quasi-isomorphe au complexe Higgs $(H / R)$.

Corollaire 5.7. Il existe un isomorphisme ("de Cartier q-déformé") de R-modules

$$
C_{q}: \mathrm{H}^{i}(q-\mathrm{DR}(A / R)) \stackrel{\sim}{\rightarrow} \Omega_{A^{\prime} / R}^{i}
$$

pour tout $i$.

Un suivi des différents morphismes permet de vérifier qu'il s'identifie bien à celui donné dans [16], Prop. 3.4, (iii) pour l'exemple $R=\mathscr{O}_{K}$ de 3.1 .

5.8. La condition de $q$-divisibilité de 4.1 garde un sens lorsque $p$ est remplacé par une puissance de $p$ et l'hypothèse de $q$-divisibilité de $R$ correspondante est cruciale pour généraliser à ce cadre les principaux résultats ci-dessus (cf. [8]). On notera ici qu'elle n'est, en général, pas vérifiée pour $R=\mathscr{O}_{K}$ et $q \neq 1$ une racine $p^{n}$-ième $(n>1)$ de l'unité comme dans 3.1.

\section{Questions-Travaux en cours}

6.1. Pour ce qui est du lien avec [4], les questions que nous nous posons sont toutes celles motivées par l'espoir suivant, dont les termes seront précisés le moment venu :

L'équivalence de catégories 5.4 est un corollaire de l'explicitation locale d'une équivalence canonique, compatible (à torsion près en général) au passage à la cohomologie, entre une catégorie convenable de cristaux sur un site q-cristallin ([4], 16.2) et une autre de cristaux sur un site prismatique ([4], 4.1).

Indépendamment de [4], une première étape pourrait consister à reformuler 5.4 comme un cas particulier d'une équivalence entre des catégories de D-modules $q$-déformés convenables, le modèle "non q-déformé" étant le point de vue proposé par Shiho ([17], Thm. 3.1) consistant à voir la correspondance d'Ogus et Vologodsky comme cas particulier d'un résultat plus général. Pour ce faire, il devrait être utile d'introduire (suivant les mêmes lignes que celles utilisées pour définir (3.8.3)) un anneau d'opérateurs différentiels q-tordus de niveau -1 (avec q "générique") déformant celui introduit par Shiho ([17], §2) et intervenant dans sa généralisation de [13].

Ensuite, dans une seconde étape, pour faire le lien entre [4] et nos constructions, l'idée la plus naturelle est de généraliser la classique équivalence entre catégories de cristaux et catégories de $\mathscr{D}$-modules et sa compatibilité au passage à la cohomologie au cadre des sites évoqués ci-dessus et des anneaux d'opérateurs différentiels $q$-déformés qui leur correspondent.

Enfin, il restera, dans une dernière étape, à définir dans un cadre géométrique non nécessairement "local" le foncteur canonique entre cristaux qu'on espère pouvoir s'expliciter comme "Frobenius divisé" au niveau des algèbres d'opérateurs différentiels $q$-déformés de niveau 0 et -1 . Ce foncteur 
devrait simplement être celui induit par le morphisme image inverse déduit du morphisme de sites décrit dans ([4], début de la preuve du Thm. 16.17).

6.2. Donnons, en conservant les notations de 3.1 et en supposant que $R$ modulo $(p)_{q}$ soit $q$ divisible, quelques indications sur la première étape et sur la définition de l'anneau $\mathrm{D}_{A, \sigma}^{(-1)}$ d'opérateurs différentiels $q$-tordus de niveau -1 (d'autres niveaux négatifs, comme dans [17], sont évidemment possibles). La définition de $\mathrm{D}_{A, \sigma}^{(-1)}$ suit celle de $\mathrm{D}_{A, \sigma}^{(0)}(3.8 .4)$ en remplaçant formellement partout $A\langle\xi\rangle_{q, y}$ par $A\left\langle\frac{\xi}{(p)_{q}}\right\rangle_{q^{p}, y}$ et en modifiant en conséquence 3.4, etc. On montre alors que la donnée d'une structure de $\mathrm{D}_{A, \sigma}^{(-1)}$-module sur un $A$-module $M$ est équivalente à la donnée d'une $\sigma^{p}$-dérivation de niveau -1, i.e. (comparer avec 5.2) d'une application $R$-linéaire $\partial_{\sigma^{p}, M}^{<1>1}: M \rightarrow M$ telle que, pour tous $r \in A, m \in M$, on ait.

$$
\partial_{\sigma^{p}, M}^{<1>1}(r m)=(p)_{q} \partial_{\sigma^{p}}(r) m+\sigma^{p}(r) \partial_{\sigma^{p}, M}^{<1>1}(m) .
$$

Il est facile de voir qu'il existe un foncteur "image inverse par Frobenius (relatif)", analogue $q$ déformé de ([17], Thm. 3.1), de la catégorie des $\mathrm{D}_{A^{\prime}, \sigma^{(}}^{(-1)}$-modules dans celle des $\mathrm{D}_{A, \sigma^{-}}^{(0)}$-modules dont nous pensons savoir démontrer ([9]) que c'est une équivalence de catégories sur les objets quasinilpotents.

En particulier, lorsque $q^{p}=1$, un $\mathrm{D}_{A^{\prime}, \sigma}^{(-1)}$-module $M$ n'est pas autre chose qu'un $A^{\prime}$-module de Higgs et le théorème 5.4 serait alors un cas particulier de cette équivalence de catégories plus générale.

6.3. Reprenons les notations de 3.1 et supposons de plus que $R$ soit une algèbre au-dessus de $\mathbb{Z}_{p}[[q-1]]$ munie d'une structure de $\delta$-anneau ([4], Def. 2.1) telle que $\delta(q)=0$ (comme pour $\left.\mathbb{Z}_{p}[[q-1]]\right)$. Supposons également $A$ munie d'une structure de $\delta$-R-algèbre telle que $\delta(x)=0$, structure qu'on étendra à $A[\xi]$ en posant

$$
\delta(\xi)=\sum_{1 \leq i \leq p-1} \frac{1}{p}\left(\begin{array}{c}
p \\
i
\end{array}\right) x^{p-i} \xi^{i} .
$$

Le premier site qui nous intéresse dans 6.1 est le site $q$-cristallin ${ }^{1}([4], 16.2)$ de $A /(q-1)$ relativement à $(R,(q-1))$. Le point crucial dans la seconde étape espérée dans 6.1 est une identification de $\left(A\langle\xi\rangle_{q, y}, I^{[1]_{q, y}}\right)(3.3)$ avec la $q$-PD-enveloppe ([4], Lemma 16.10) de $(A[\xi],(\xi))$. Précisons le résultat auquel nous parvenons. Pour définition d'une q-PD-paire $(B, J)$ (comparer [4], Def. 16.2) ne retenons ici (cf. $\left.{ }^{1}\right)$ que la donnée d'une $\delta$-algèbre $B$ au-dessus de $(R, \delta)$, sans $(p)_{p}$-torsion, munie d'un idéal $J$ tel que $\phi(J) \subset(p)_{q} B$ avec $\phi(b):=b^{p}+p \delta(b)$ pour tout $b \in B$. Si $C$ est une $\delta$-R-algèbre et $I$ un idéal quelconque de $C$ (auquel cas, on dira que $(C, I)$ est une $\delta$-paire, cf. ([4], Def. 3.2)), sa $q$-PD-enveloppe, notée $\left(C^{[]}, I^{[]}\right)$, est pour nous ici la $q$-PD-paire universelle (dont la proposition ci-dessous prouve, pour le cas qui la concerne, l'existence et l'unicité à isomorphisme près) pour le prolongement (unique) à $\left(C^{[]}, I^{[]}\right)$de tout morphisme $(C, I) \rightarrow(B, J)$ d'une $\delta$-paire dans une $q$-PD-paire. On a alors la

Proposition 6.4 ([9]). Si $A$ est une $\delta$-R-algèbre sans $(p)_{q}$-torsion, alors $\left(A\langle\xi\rangle_{q, y}, I^{[1]_{q, y}}\right)(3.3)$ s'identifie à la q-PD-enveloppe ${ }^{1}$ de la $\delta$-paire $(A[\xi],(\xi))$.

1. CAVEAT : Nous empruntons ici et plus bas, abusivement, la terminologie de [4] mais ignorons dans nos rappels certaines des propriétés additionnelles sur les objets requises dans loc. cit. si elles ne jouent pas de rôle dans ce que l'on veut expliquer ici (voir d'ailleurs, à ce sujet, les commentaires sur leur éventuel caractère provisoire sous la définition 16.2 de [4]). Les ajustements précis avec les hypothèses de [4], particulièrement ceux nécessitant de prendre en compte complétions et topologies (ne serait-ce que dans la définition des sites) seront donnés dans [9]. 
La démonstration consiste à se ramener au cas $R=\mathbb{Z}_{p}[[q-1]]$ et $A=R[x]$ puis, utilisant l'écriture $p$-adique de $n=\sum_{r \geq 0} k_{r} p^{r}$, de montrer que les $v_{n}:=\xi^{k_{0}} \prod_{r \geq 0}\left(\delta^{r}([\phi](\xi))\right)^{k_{r}+1}$ forment, pour $n \in \mathbb{N}$, une base, comme $A$-module, de $A\langle\xi\rangle_{q, y}$. Ici

$$
[\phi]: A\langle\xi\rangle_{q, y} \rightarrow A\langle\xi\rangle_{q, y} ;[\phi]\left(\xi^{[n]_{q, y}}\right)=\sum_{i=n}^{p n} B_{n, i}(q) x^{p n-i} \xi^{[i]_{q, y}}
$$

tient compte, par rapport à [F*] (4.6.1), de l'usage du Frobenius absolu dans [4] plutôt que relatif dans [8] . Si maintenant $(B, J)$ est une $q$-PD-paire, tout morphisme de $\delta$-paires $u:(A[\xi],(\xi)) \rightarrow$ $(B, J)$ s'étend alors uniquement à $A\langle\xi\rangle_{q, y}$ par un morphisme d'anneaux envoyant $v_{n} \in A\langle\xi\rangle_{q, y}$ sur $f^{k_{0}} \prod_{r \geq 0}\left(\delta^{r}(g)\right)^{k_{r}+1} \in B$ avec $f:=u(\xi)$ et $g \in B$ unique tel que $\phi(f)=(p)_{q} g$.

Signalons que le cas $q=1$ est celui traité dans ([4], Lem. 2.35) et, pour le lecteur intéressé, l'existence d'un analogue ([15], Lem. 1.3), au moins lorsque $q-1 \in R^{\times}$, pour les $\lambda$-anneaux.

6.5. Conservons les notations de 6.3 et supposons que $\left(R,(p)_{q}\right)$ soit un prisme borné ([4], Def. 3.2) pour pouvoir réfèrer à [4]. Notons $A^{(1)}$ le quotient $A^{\prime} /(p)_{q} A^{\prime}$. Le second site qui nous intéresse dans 6.1 est le site prismatique de $A^{(1)}$ relativement à $\left(R,\left((p)_{q}\right)\right)$. Comme dans 6.3 , le point crucial dans la seconde étape espérée dans 6.1 est de disposer d'une description adéquate (que nous appliquerons in fine à $A^{\prime}$ plutôt qu'à $\left.A\right)$ de l'enveloppe prismatique ([4], Cor. 3.14) de $(A[\xi],(\xi))$ relativement à $\left(R,\left((p)_{q}\right)\right)$. Reprenant les termes de la construction donnée dans loc. cit., considérons donc juste ici la question du prolongement universel d'un morphisme de $\delta$-paires (au-dessus de la $\delta$ paire $\left.\left(R,\left((p)_{q}\right)\right)\right) u:(A[\xi],(\xi)) \rightarrow\left(B,\left((p)_{q}\right)\right)$ avec $B$ sans $(p)_{q}$-torsion à une $\delta$-paire de la forme $\left(C,\left((p)_{q}\right)\right)$. Nous montrons qu'un tel objet universel existe et nous l'appellerons (cf. $\left.{ }^{1}\right)$ dans la proposition qui suit enveloppe prismatique de $(A[\xi],(\xi))$.

Proposition 6.6 ([9]). Si A est une $\delta$-R-algèbre sans $(p)_{q}$-torsion, alors $\left(A\left\langle\frac{\xi}{(p)_{q}}\right\rangle_{q^{p}, y},\left((p)_{q}\right)\right)(6.2)$ s'identifie à l'enveloppe prismatique ${ }^{1}$ de la $\delta$-paire $(A[\xi],(\xi))$.

En effet, la variante de (4.4.1) utilisant le Frobenius absolu de $A$ plutôt que relatif comme dans [8] fournit une application (dont (6.4.1) est la variante "divisée")

$$
\phi: A\langle\xi\rangle_{q, y} \rightarrow A\langle\xi\rangle_{q, y} ; \phi\left(\xi^{[n]_{q, y}}\right)=\sum_{i=n}^{p n} A_{n, i}(q) x^{p n-i} \xi^{[i]_{q, y}} .
$$

L'équation (4.5.2) suffit alors à voir (rappelons au passage que $\phi(q)=q^{p}$ ) que $A\left\langle\frac{\xi}{(p)_{q}}\right\rangle_{q^{p}, y}$ est bien muni d'un relèvement de Frobenius et, par suite, d'une structure de $\delta$-anneau. Enfin, le même argument que dans 6.4 (avec $\left.u(\xi)=(p)_{q} g\right)$ donne le prolongement cherché de $u:(A[\xi],(\xi)) \rightarrow$ $\left(B,(p)_{q} B\right)$ à $\left(A\left\langle\frac{\xi}{(p)_{q}}\right\rangle_{q^{p}, y},\left((p)_{q}\right)\right)$.

6.7. Pour terminer, remarquons que les arguments de Shiho ([17]) ne nécessitaient pas d'interprétation de ses $\mathrm{D}^{(-1)}$-modules (loc. cit $\S 2$ ) quasi-nilpotents en termes de cristaux sur un site mais que, lorsque $q=1$, le site prismatique $([4], 4.1)$ en fournit une, qui dans ce cas particulier est juste une variante "avec $\delta$-structures" de celle déjà établie dans ([14], Def. 1.3.1, [19], Def. 7.1) (l'ajout de $\delta$-structures évitant précisément les puissances divisées additionnelles sur les anneaux d'opérateurs différentiels considérés des ces articles). Enfin, compte tenu des considérations topologiques délicates à développer sur les sites considérés dans 6.3-6.5 nous laissons pour ailleurs la discussion d'une possible approche alternative à l'équivalence cherchée dans 6.1 qui serait l'analogue de ([14], Thm. 1.4.3) (équivalence de topos). 


\section{RÉFÉRENCES}

[1] A. Abbes, M. Gros, T. Tsuji, The p-adic Simpson correspondence. Annals of Math. Stud., 193, Princeton Univ. Press (2016). [Cité à la page 2.]

[2] P. Berthelot, D-modules arithmétiques. I. Opérateurs différentiels de niveau fini. Ann. Sc. de l'É.N.S, 4 ième série, t. 29, n. 2 (1996), 185-272. [Cité à la page 3.]

[3] B. Bhatt, M. Morrow, P. Scholze, Integral p-adic Hodge theory. Publ. Math. Inst. Hautes Études Sci. 128 (2018), 219-397. [Cité aux pages 2 et 6.]

[4] B. Bhatt, P. Scholze, Prisms and prismatic cohomology. arXiv :1905.08229v2 (2019). [Cité aux pages 2, 3, 11, 12, et 13.]

[5] P. Deligne, L. Illusie, Relèvements modulo $p^{2}$ et décomposition du complexe de de Rham. Inv. Math. 89 (1987), 247-270. [Cité aux pages 1 et 5.]

[6] M. Gros, , B. Le Stum, A. Quirós, A Simpson correspondence in positive characteristic. Pub. RIMS Kyoto Univ. 46 (2010), 1-35. [Cité aux pages 2, 3, 4, 5, 6, 9, et 10.]

[7] M. Gros, , B. Le Stum, Une neutralisation explicite de l'algèbre de Weyl quantique complétée. Comm. Algebra 42 no. 5, (2014), 2163-2170. [Cité aux pages 7 et 10.]

[8] M. Gros, , B. Le Stum, A. Quirós, Twisted divided powers and applications. J. Number Theory (2019), (https ://doi.org/10.1016/j-jnt.2019.02.009). [Cité aux pages 2, 7, 8, 9, 10, 11, et 13.]

[9] M. Gros, , B. Le Stum, A. Quirós, En préparation. [Cité aux pages 12 et 13.]

[10] N. Katz, Nilpotent connections and the monodromy theorem : applications of a result of Turrittin. Publ. Math. IHÉS 39 (1970), 175-232. [Cité aux pages 1 et 5.]

[11] B. Le Stum, A. Quirós, Twisted calculus. Communications in Algebra, 46 :12 (2018), 5290-5319. (https ://doi.org/10.1080/00927872.2018.1464168). [Cité à la page 11.]

[12] B. Le Stum, A. Quirós, Formal confluence of quantum differential operators. Pacific Journal of Mathematics 292(2), (2018), 427-478. (https ://doi.org/10.2140/pjm.2018.292.427). [Cité à la page 8.]

[13] A. Ogus, V. Vologodsky, Non abelian Hodge theory in characteristic p. Publ. Math. IHÉS 101 (2007), 1-138. [Cité aux pages 1, 2, 4, et 11.]

[14] H. Oyama, PD Higgs crystals and Higgs cohomology in characteristic p. J. Algebraic Geom. 26 (2017), 735-802. [Cité aux pages 2 et 13.]

[15] J. P. Pridham, On q-De Rham cohomology via $\Lambda$-rings. Math. Annalen (https ://doi.org/10.1007/s00208019-01806-7). [Cité aux pages 2 et 13.]

[16] P. Scholze, Canonical q-deformations in arithmetic geometry. Ann. Fac. Sci. Toulouse Math. (6) 26 (2017), no. 5, 1163-1192. [Cité aux pages 2, 6, 7, 10, et 11.]

[17] A. Sнiно, Notes on generalizations of local Ogus-Vologodsky correspondence. J. Math. Sci. Univ. Tokyo 22 (2015), 793-875. [Cité aux pages 2, 11, 12, et 13.]

[18] T. Tsuji, Exposé du 12 Mai 2017 au Simons Symposium on p-adic Hodge theory et articles en préparation dont un en collaboration avec Matthew Morrow. [Cité à la page 2.]

[19] D. Xu, Lifting the Cartier transform of Ogus-Vologodsky modulo $p^{n}$. Mémoires de la Société Mathématique de France, t. 163 (2019). [Cité aux pages 2 et 13.]

M.G. CNRS UmR 6625, irmar, Université de Rennes 1, Campus de Beaulieu, 35042 Rennes cedex, FRANCE

E-mail address: michel.gros@univ-rennes1.fr 